\title{
VEHICLE YAW MOTION CONTROL USING TAKAGI-SUGENO MODELING AND QUADRATIC BOUNDEDNESS VIA DYNAMIC OUTPUT FEEDBACK
}

\author{
Saïd Mammar* \\ Lydie Nouvelière \\ IBISC - FRE CNRS 3190 \\ Evry, 91020 Cedex \\ France \\ Email: said.mammar@ibisc.univ-evry.fr
}

\author{
Nicoleta Minoiu-Enache \\ Sébastien Glaser \\ Benoit Lusetti \\ Dominique Gruyer \\ LIVIC LCPC-INRETS \\ Versailles Satory, 7800 \\ France
}

\begin{abstract}
This paper presents the design and the simulation test of a Takagi-Sugeno (TS) fuzzy output feedback for yaw motion control. An integrated steering and differential braking controller based on invariant sets, quadratic boundedness theory and a common Lyapunov function has been developed. The TS fuzzy model is able to handle elegantly the nonlinear behavior the vehicle lateral dynamics. The computation of the control law has been achieved using Linear and Bilinear Matrix Inequalities (LMI-BMI) methods. Simulation test shows the controlled car is able to achieve the ISO3888-2 transient maneuver. Some design parameters can be adjusted to handle the tradeoff between safety constraints and comfort specifications.
\end{abstract}

\section{NOMENCLATURE}

$m$ Vehicle total mass $1600 \mathrm{~kg}$.

$c_{f 0} \quad$ Front cornering stiffness $40000 \mathrm{~N} / \mathrm{rad}$.

$c_{r 0} \quad$ Rear cornering stiffness $35000 \mathrm{~N} / \mathrm{rad}$.

$J$ Vehicle yaw moment of inertia $2454 \mathrm{~kg} \cdot \mathrm{m}^{2}$.

$l_{f}$ Distance form CG to front axle $1.22 \mathrm{~m}$.

$l_{r}$ Distance from CG to rear axle $1.44 m$.

$v$ Longitudinal velocity.

$\mu \quad$ Road adhesion coefficient $[0,1]$.

\section{INTRODUCTION}

There is no doubt that electronic stability control systems (ESC) have largely contributed to accident and death reduction during this last decade [18]. Vehicle loss of control is the main accident situation which is particularly well addressed by ESC. In fact, it has been proven to enhance vehicle directional stability through yaw torque generated by applying suitable wheel braking. In addition to vehicle loss of control, ESC is potentially highly effective in preventing run-off road crashes, rollover accident and multi-vehicle crashes. However, further reduction of these types of accidents remains a major goal of car manufacturers and suppliers since they represent a high percentage of the overall accident rate and the number of deaths [18].

Starting with the original concept introduced by Bosch in 1995 [19] [20], different approaches have been developed for ESC. Today's systems act on the vehicle lateral dynamics mainly through independent wheel braking. Beyond this solution, recent studies have demonstrated that differential braking may have a better effect on yaw dynamics than independent active wheel braking [21]. Optimal strategies for braking forces allocation have been explored in [22]. In parallel, vehicle handling has been also investigated through active steering which allows to act as an interface between the driver steering wheel and the tires [9], [12]. Even if the mechanical linkage between them is still a limiting factor, solutions have been already implemented in series pro-

\footnotetext{
*Address all correspondence to this author.
} 
duction. For example, a concept of advanced front steering [26] allows to add a limited steering angle which is able to generate sufficient lateral forces for lateral disturbance rejection [14], [23]. However, this type of solution is mainly reserved for driving situations characterized by a low sideslip angle [25], [12] and real gain from active steering will come with steer-by-wire systems which will offer additional freedom-factors for controller intervention [24].

In this paper, an integrated control strategy which combines active steering and differential braking and uses a dynamic fuzzy output feedback concept is proposed. Takagi-Sugeno fuzzy systems offer an elegant approach for modeling non linear systems in a wide operating domain. In the literature, control of fuzzy systems has been mainly addressed using parallel distributed control (PDC) concept [6], [15], [5], [7]. State feedback controllers have been first designed with different performance index. When the state is unmeasurable, a fuzzy state observer could be incorporated into the controller design in order to form an output feedback fuzzy control. The fuzzy observer generally uses the same rules as that of the state feedback PDC rules [10]. Sufficient conditions for PDC static output feedback have been also developed for nominal and uncertain fuzzy systems [8]. The dynamic output feedback case has been considered in recent work. The dynamic output feedback formulation considered in this paper presents two main advantages: better flexibility to formulate the stabilization conditions and ability to handle input or state constraints and bounded disturbances. This controller uses the property of quadratic boundedness and invariant set [4].

In fact, during control intervention, it is important to ensure a good safety level. To achieve this, it has been chosen to build an invariant set for the system state and to require that each trajectory that starts inside the invariant set will not exceed it, hence the trajectories will be bounded inside it.

The nonlinear vehicle dynamics [13] are first modeled using a discrete tile 4 rules Takagi-Sugeno fuzzy model based on the tire sideslip angles thresholds [15], [11]. In addition, the model is developed in such a way to include the error dynamics of the yaw rate reference model following. Afterwards, a dynamic control fuzzy output feedback is synthesized [4], [8]. It handles both input and state constraints using only measurements of the yaw rate and the steering angle [16].

The next Section gives a description of the developed vehicle lateral dynamics Takagi-Sugeno model of the vehicle. The fuzzy output feedback synthesis, including the requirements concerning the quadratic boundedness, the state constraints and control limitation are then presented. Simulation results for maneuvers which excite the nonlinear tire dynamics are provided. The conclusions wrap up the paper.

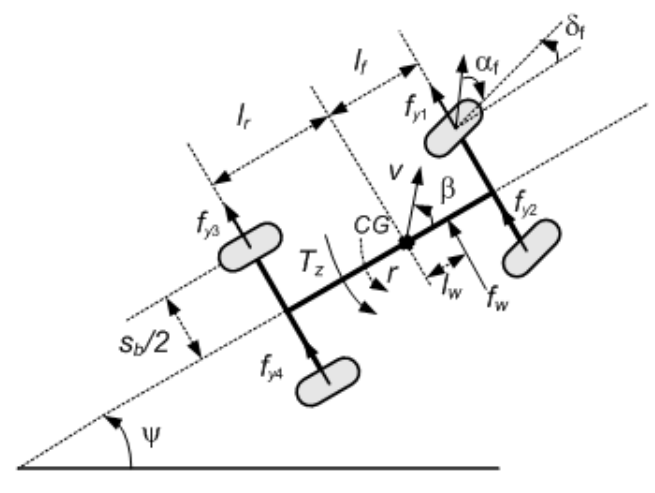

(a)

FIGURE 1. Vehicle model

\section{VEHICLE LATERAL DYNAMICS T-S MODEL}

As we are concerned with lateral control, a simple nonlinear model of a vehicle is obtained by neglecting the roll and pitch motions. This model includes the lateral translational motion and the yaw motion (Figure 1). The two wheels of each axle are lumped into one located at its center. This leads to the vehicle bicycle model. The lateral forces between the each tire and the road surface are added at each axle leading to two resulting forces $f_{f}\left(\alpha_{f}\right)$ and $f_{r}\left(\alpha_{r}\right)$ at the front and rear wheels of the bicycle model respectively. These forces which will be detailed below are function of the front and rear tires sideslip angle, denoted $\alpha_{f}$ and $\alpha_{r}$ respectively.

The lateral translation and rotational yaw motion equations written in the vehicle fixed frame take the following form

$$
\left[\begin{array}{c}
m v(\dot{\beta}+r) \\
J \dot{r}
\end{array}\right]=\left[\begin{array}{ccc}
1 & 1 & 0 \\
l_{f} & -l_{r} & 1
\end{array}\right]\left[\begin{array}{c}
f_{f}\left(\alpha_{f}\right) \\
f_{r}\left(\alpha_{r}\right) \\
T_{z}
\end{array}\right]
$$

where $\beta$ is the vehicle side slip angle, $r$ is the yaw rate and $T_{z}$ is the yaw moment input applied by differential wheel braking. $m$ is the vehicle mass while $J$ is the vehicle moment of inertia. The vehicle center of gravity is located at a distance $l_{f}$ from the front axle and a distance $l_{r}$ from the rear axle.

Assuming that the angles remain small, the front and the rear sideslip angles are given by:

$$
\begin{aligned}
& \alpha_{f}=\delta_{f}-\left(\beta+\frac{l_{f}}{v} r\right) \\
& \alpha_{r}=-\beta+\frac{l_{r}}{v} r
\end{aligned}
$$




\section{Lateral tire forces model}

Several types of models of the forces of tire-pavement interaction have been proposed in the literature [13]. They are usually derived from experimental data, as for the Pacejka model, and have as parameters the adhesion, the speed $v$ and the normal force $f_{n i}$. The shape of the lateral force is often similar from one model to another. A first linear domain for small sideslip angle allows to define a slope factor $c_{y_{i}}$ called the tire cornering stiffness coefficient. When the sideslip angle increases, the tire enters a nonlinear operating zone where the lateral force saturates. The maximum value defines the limit of the vehicle maneuverability, resulting in a loss of controllability that can cause an understeering phenomenon or an unusual oversteering which may surprise the driver. In the sequel, the model HSRI ${ }^{1}$ will be used [17]. It allows a simple lateral tire forces formulation which integrates two of the cited parameters, the adhesion $\mu$ and the normal force $f_{n_{i}}$ :

$$
f_{y_{i}}=c_{y_{i}} f(\lambda) \tan \alpha_{i}
$$

where

$$
\lambda=\frac{\mu f_{n_{i}}}{2 c_{y_{i}}\left|\tan \alpha_{i}\right|}
$$

and

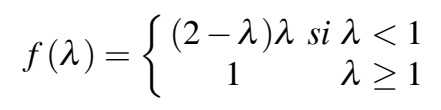

Let note first that the HSRI model is characterized by the following properties:

The slope at the origin is a function of the adhesion.

The maximum force value that can be mobilized is $f_{n_{i}}$.

For maximum adhesion, the slope at the origin intersects the saturation value for the tire sideslip angle value $\alpha_{s a t}=\frac{f_{n_{i}}}{c_{y_{i}}}$.

The goal now is to achieve a Takagi-Sugeno fuzzy model which covers the entire operating domain (linear and nonlinear) of the forces [1]. The developed process is naturally applicable for any other type of tire forces model. Similarly, even if triangular membership functions are used, any other membership functions could be used. The definition and the value of the above parameters are described in the nomenclature.

\section{Four rules Takagi-Sugeno vehicle fuzzy model}

The nonlinear vehicle model is transformed into a four rules Takagi-Sugeno (T-S) fuzzy model according to the values of the front and rear cornering stiffnesses:

\footnotetext{
${ }^{1}$ Highway Safety Research Institute, United-States
}

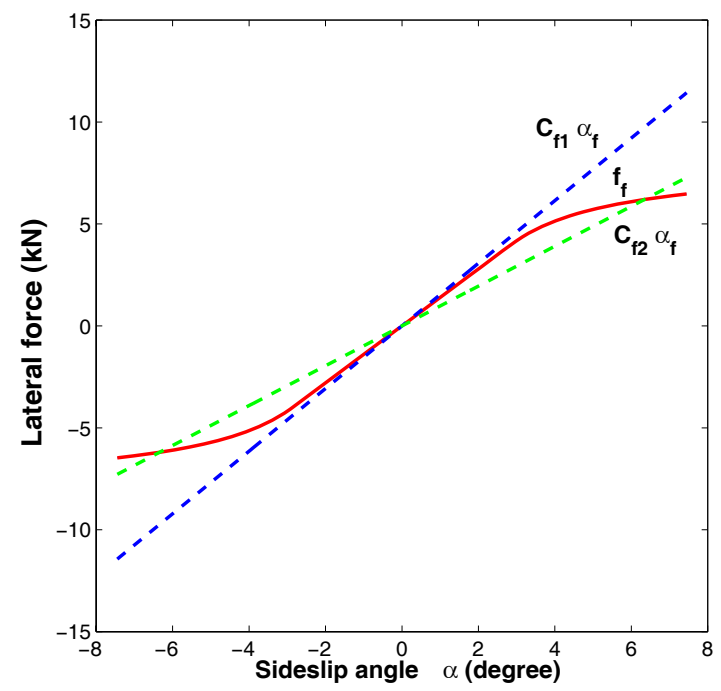

FIGURE 2. Tire lateral force given by the HSRI model and sector based approximation.

$$
\begin{aligned}
& \text { if }\left|\alpha_{f}\right| \text { is } m_{1} \text { and }\left|\alpha_{r}\right| \text { is } n_{1} \text { then }\left\{\begin{array}{l}
f_{f}=c_{f_{1}} \alpha_{f} \\
f_{r}=c_{r_{1}} \alpha_{r}
\end{array}\right. \\
& \text { if }\left|\alpha_{f}\right| \text { is } m_{2} \text { and }\left|\alpha_{r}\right| \text { is } n_{1} \text { then }\left\{\begin{array}{l}
f_{f}=c_{f_{2}} \alpha_{f} \\
f_{r}=c_{r_{1}} \alpha_{r}
\end{array}\right. \\
& \text { if }\left|\alpha_{f}\right| \text { is } m_{1} \text { and }\left|\alpha_{r}\right| \text { is } n_{2} \text { then }\left\{\begin{array}{l}
f_{f}=c_{f_{1}} \alpha_{f} \\
f_{r}=c_{r_{2}} \alpha_{r}
\end{array}\right. \\
& \text { if }\left|\alpha_{f}\right| \text { is } m_{2} \text { and }\left|\alpha_{r}\right| \text { is } n_{2} \text { then }\left\{\begin{array}{l}
f_{f}=c_{f_{2}} \alpha_{f} \\
f_{r}=c_{r_{2}} \alpha_{r}
\end{array}\right.
\end{aligned}
$$

The membership functions $m_{i}$ and $n_{i}(i=1,2)$ are determined by the approximation method of nonlinear function by linear sectors. Coefficients $c_{f_{i}}$ and $c_{r_{i}}(i=1,2)$ represent the tire cornering stiffnesses associated to each sector. In fact they represent also the slope of the limits of the sectors which include the tire forces (Fig. 2). For example, given two coefficients $c_{f_{1}}$ and $c_{f_{2}}$, chosen according to the expected road adhesion and driving conditions, one can determine the membership functions $m_{1}\left(\alpha_{f}\right)$ and $m_{2}\left(\alpha_{f}\right)$ using the following set of equations:

$$
\left\{\begin{array}{l}
\left(c_{f_{1}} m_{1}\left(\alpha_{f}\right)+c_{f_{2}} m_{2}\left(\alpha_{f}\right)\right) \alpha_{f}=f_{f}\left(\alpha_{f}\right) \\
m_{2}=1-m_{1}
\end{array}\right.
$$

which leads to:

$$
m_{1}=\frac{f_{f}-c_{f_{2}} \alpha_{f}}{\left(c_{f_{1}}-c_{f_{2}}\right) \alpha_{f}}
$$

The evolution of the two functions $m_{1}$ and $m_{2}$ as functions of the sideslip angle are shown in Figure 3. They are obtained with 
numerical values: $c_{f_{1}}=1.1 c_{f}$ and $c_{f_{2}}=0.7 c_{f}$. It is important to outline that this sector representation is an exact approximation of the non linear system.

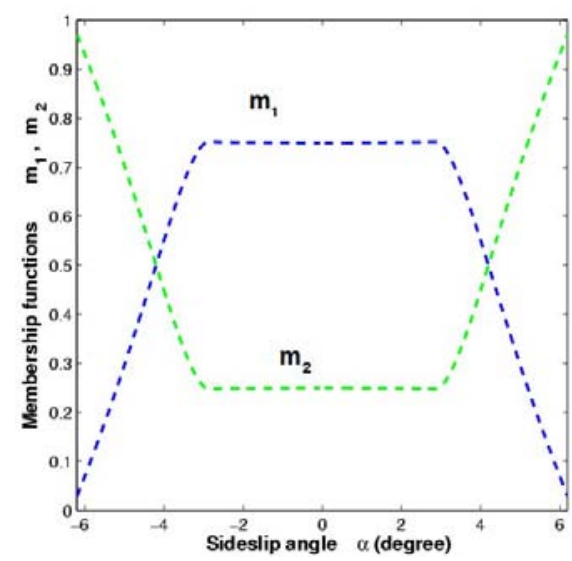

FIGURE 3. Membership functions $m_{1}$ and $m_{2}$ associated to the front tire contact forces.

The membership functions $n_{1}$ and $n_{2}$ for the rear tire forces are obtained by the same procedure. Finally, one can write:

$$
\left\{\begin{aligned}
f_{f} & =\left[\left(h_{1}+h_{3}\right) c_{f_{1}}+\left(h_{2}+h_{4}\right) c_{f_{2}}\right] \alpha_{f} \\
f_{r} & =\left[\left(h_{1}+h_{2}\right) c_{r_{1}}+\left(h_{3}+h_{4}\right) c_{r_{2}}\right] \alpha_{r}
\end{aligned}\right.
$$

with $h_{1}=m_{1} \times n_{1}, h_{2}=m_{2} \times n_{1}, h_{3}=m_{1} \times n_{2}$ and $h_{4}=m_{2} \times n_{2}$.

In order to have the front and the rear sideslip angle as state vector components, let us define the state $\bar{x}=\left[\alpha_{f}, \alpha_{r}, \delta_{f}\right]^{T}$ and the control input $u=\left[\dot{\delta}_{f}, T_{z}\right]^{T}$, the fuzzy system takes the form:

$$
\bar{x}=\sum_{i=1}^{4} h_{i}\left(\alpha_{f}, \alpha_{r}\right) \overline{A_{i}} \bar{x}+\bar{B} u
$$

where

$$
\overline{A_{i}}=\left[\begin{array}{ccc}
a_{11 i} & a_{12 i} & a_{13} \\
a_{21 i} & a_{22 i} & a_{23} \\
0 & 0 & 0
\end{array}\right], \bar{B}=\left[\begin{array}{cc}
1 & -\frac{l_{f}}{J_{v}} \\
0 & \frac{l_{r}}{J_{v}} \\
1 & 0
\end{array}\right]
$$

where

$$
\left\{\begin{array}{l}
a_{11 i}=-\frac{v}{l_{f}+l_{r}}-\frac{1}{v}\left(\frac{1}{m}+\frac{l_{r} l_{f}}{I_{z}}\right) c_{f i}, \\
a_{12 i}=\frac{v}{l_{f}+l_{r}}-\frac{1}{v}\left(\frac{1}{m}-\frac{l_{f} l_{r}}{I_{z}}\right) c_{r_{i}}, \\
a_{21 i}=-\frac{v}{l_{f}+l_{r}}-\frac{1}{v}\left(\frac{1}{m}-\frac{l_{r}^{2}}{I_{z}}\right) c_{f i}, \\
a_{22 i}=\frac{v}{l_{f}+l_{r}}-\frac{1}{v}\left(\frac{1}{m}+\frac{l_{r}^{2}}{I_{z}}\right) c_{r_{i}}, \\
a_{13}=\frac{v}{l_{f}+l_{r}}, \\
a_{23}=\frac{v}{l_{f}+l_{r}} .
\end{array}\right.
$$

\section{Reference yaw rate tracking}

Ideally, the vehicle should respond to driver's steering angle as a speed depended yaw rate reference steady state value with almost constant settling time. Let $T_{0}$ be the desired transfer function between $\delta_{o}$ and $r$. In order to ensure at nominal speed, the same steady state value for the controlled and the conventional car, the reference model is chosen as a first order transfer function with the same steady state gain as the conventional car. It is of the form $r_{d}=\frac{K_{d}(v)}{\tau s+1} \delta_{d}$. The speed dependent steady state gain is $K_{d}(v)$ and $\tau=0.2 \mathrm{sec}$.

In order to ensure that the yaw rate reference value is achieved in steady state, the integral $z$ of the yaw rate tracking error is added as state a variable:

$$
\dot{z}=r-r_{d}=\frac{\delta_{f}+\alpha_{r}-\alpha_{f}}{l_{f}+l_{r}} v-r_{d}
$$

This variable is thus added to the previous third order model while the desired yaw rate is considered as a disturbance. The fuzzy model is finally discretized at a sample time of $0.005 \mathrm{sec}$ using a Tustin's method. The final fuzzy model is of the form:

$$
\begin{aligned}
& x(t+1)=\sum_{i=1}^{4} h_{i}\left(\alpha_{f}, \alpha_{r}\right) A_{i} x(t)+B u(t)+E w(t) \\
& y(t)=C x(t)+D w(t)
\end{aligned}
$$

where $x=\left[\alpha_{f}, \alpha_{r}, \delta_{f}, z\right]^{T}$ and $y(t)=[r, z]^{T}$. The disturbance $w(t)=r_{d}(t) \in \varepsilon_{Q}=\left\{w \in \mathscr{R} / w^{T} Q w \leq 1\right\}$ is bounded. Matrices $A_{i}$ and $B$ can be easily derived from equations (8) and (9). This discrete time fuzzy system is characterized by common $B$, $E$ and $C$ matrices for all the sub-models:

$$
\begin{aligned}
& E=\left[\begin{array}{llll}
0 & 0 & 0 & -1
\end{array}\right]^{T} \\
& C=\left[\begin{array}{cccc}
\frac{-v}{l_{f}+l_{r}} v & \frac{v}{l_{f}+l_{r}} & \frac{v}{l_{f}+l_{r}} & 0 \\
0 & 0 & 0 & 1
\end{array}\right]
\end{aligned}
$$

This property simplifies drastically the stability and performance conditions as only simple summations are involved. The matrix $D$ is zero for the nominal system. 


\section{DYNAMIC OUTPUT FEEDBACK FUZZY CONTROLLER} form:

In the following, we seek a dynamic fuzzy controller of the

$$
\begin{aligned}
& x_{c}(t+1)=\sum_{i=1}^{4} h_{i}\left(\alpha_{f}, \alpha_{r}\right) A_{c}^{i} x_{c}(t)+B_{c} y(t) \\
& u(t)=C_{c} x_{c}(t)+D_{c} y(t)
\end{aligned}
$$

where $x_{c} \in \mathscr{R}^{5}$ is the controller state; $\left\{A_{c}^{i}, B_{c}, C_{c}, D_{c}\right\}$ are matrices to be designed.

This controller still uses the parallel distributed compensation (PDC) concept of the fuzzy system control. In this concept, each control rule is distributively designed for the corresponding rule of a T-S fuzzy model. Linear control theory can then be used to design controllers for each of the consequent part of the fuzzy system while ensuring the same properties for the fuzzy system.

As pointed out in [4], $D_{c}$ is an important parameter for stabilization, and the controller structure is able to handle constraints on the input and the state. By combining (10) and (11), the augmented closed-loop fuzzy model is given by

$$
\tilde{x}(t+1)=\sum_{i=1}^{4} h_{i}\left(\alpha_{f}, \alpha_{r}\right) \Phi_{i} \tilde{x}(t)+\Gamma w(t) .
$$

where $\tilde{x}=\left[\begin{array}{c}x \\ x_{c}\end{array}\right], \quad \Phi_{i}=\left[\begin{array}{cc}A_{i}+B D_{c} C & B C_{c} \\ B_{c} C & A_{c}^{i}\end{array}\right]$ and $\Gamma=$ $\left[\begin{array}{c}B D_{c} D+E \\ B_{c} D\end{array}\right]$.

Let $\Phi_{z}=\sum_{i=1}^{4} h_{i}\left(\alpha_{f}, \alpha_{r}\right) \Phi_{i}$, the closed loop system takes the form: $\tilde{x}(t+1)=\Phi_{z} \tilde{x}(t)+\Gamma w(t)$. Finally the control input $u(t)$ is given by:

$$
u(t)=\left[D_{c} C C_{c}\right] \tilde{x}(t)+D_{c} D w(t)
$$

\section{Invariant set and output feedback PDC control}

Assume that there exists a quadratic function $V(\tilde{x})=\tilde{x}^{T} P \tilde{x}$, where $P$ is a symmetric, positive definite matrix that satisfies the condition [2], [3]:

$$
V(\tilde{x}+1) \leq V(\tilde{x}), \forall \tilde{x}, w \text { satisfying }(12), w^{T} Q w \leq 1, V(\tilde{x}) \geq 1 .
$$

Consider the reachable set $\Lambda$ defined by:

$$
\begin{array}{r}
\Lambda \triangleq \quad\{\tilde{x}(T) \mid \tilde{x}, w \text { satisfying }(12), \\
\left.\tilde{x}(0)=0, w^{T} Q w \leq 1, T \geq 0\right\} .
\end{array}
$$

The set $\varepsilon_{P}$ is defined by:

$$
\varepsilon_{P}=\left\{\tilde{x}(t) \in \mathscr{R}^{8} \mid \tilde{x}(t)^{T} P \tilde{x}(t) \leq 1\right\},
$$

is an invariant set for the system (12) with $w \in \mathscr{R}, w^{T} Q w \leq 1$. This means that every trajectory that starts inside $\varepsilon_{P}$ remains inside it for $t \rightarrow \infty$.

The existence of such a function $V(\tilde{x})$ means that the set $\varepsilon_{P}$ is an outer approximation of the reachable set $\Lambda$.

$\varepsilon_{P}$ is also and outer approximation of the reachable set

$$
\begin{aligned}
\Lambda^{*} \triangleq & \{\tilde{x}(T) \mid \tilde{x}, w \text { satisfying equation (12), } \\
& \left.\tilde{x}(0) \in \varepsilon(P), w^{T} Q w \leq 1, T \geq 0\right\} .
\end{aligned}
$$

In this section the control law and the invariant set $\varepsilon_{P}$ are synthesized. This is achieved using BMI (Bilinear Matrix Inequalities) optimization method such that the system without the disturbance is asymptotically stable and at the same time, the reachable set for an initial state values inside the invariant set is contained in this invariant set.

\section{Invariant set - quadratic boundedness}

According to the previous considerations, the closed loop linear system $\tilde{x}(t+1)=\Phi_{z} \tilde{x}(t)+\Gamma w(t)$ is strictly quadratically bounded with a common Lyapunov matrix $P>0$ for all allowable $w(t) \in \varepsilon_{Q}$, for $t>0$, if $\tilde{x}(t)^{T} P \tilde{x}(t)>1$ implies $\left(\Phi_{z} \tilde{x}(t)+\Gamma w(t)\right)^{T} P\left(\Phi_{z} \tilde{x}(t)+\Gamma w(t)\right)<\tilde{x}^{T} P \tilde{x}$, for any $w \in \varepsilon_{Q}$.

The corresponding condition is obtained using the $S$-procedure and invoking the Schur complement, using that the satisfaction of $w \in \varepsilon_{Q}$ and $\tilde{x}^{T} P \tilde{x} \geq 1$ implies $w^{T} Q w \leq \tilde{x}^{T} P \tilde{x}$.

The implication

$$
\begin{aligned}
w^{T} Q w & \leq \tilde{x}^{T} P \tilde{x} \\
& \Longrightarrow\left(\Phi_{z} \tilde{x}(t)+\Gamma w(t)\right)^{T} P\left(\Phi_{z} \tilde{x}(t)+\Gamma w(t)\right) \leq \tilde{x}^{T} P \tilde{x}
\end{aligned}
$$

is equivalent to:

$$
\exists \alpha>0:\left[\begin{array}{ccc}
(1-\alpha) P & * & * \\
0 & \alpha Q & * \\
\Phi_{z} & \Gamma & P^{-1}
\end{array}\right] \geq 0
$$

Let define $P=\left[\begin{array}{ll}P_{1} & P_{2}^{T} \\ P_{2} & P_{3}\end{array}\right]$ and $P^{-1}=\left[\begin{array}{ll}M_{1} & M_{2}^{T} \\ M_{2} & M_{3}\end{array}\right]$. Assuming that $P_{2}$ and $M_{2}$ are full rank matrices, let us define $T_{1}=\left[\begin{array}{ll}I & M_{1} \\ 0 & M_{2}\end{array}\right]$ and $T_{2}=\left[\begin{array}{ll}I & P_{1} \\ 0 & P_{2}\end{array}\right]$. By pre- and post-multiplying the left-hand side of (18) with $\operatorname{diag}\left\{T_{1}^{T}, I, T_{2}^{T}\right\}$ and $\operatorname{diag}\left\{T_{1}, I, T_{2}\right\}$, respectively, and 
applying

$$
\left\{\begin{array}{l}
\hat{D}_{c}=D_{c} \\
\hat{C}_{c}=D_{c} C M_{1}+C_{c} M_{2} \\
\hat{B}_{c}=P_{1} B D_{c}+P_{2}^{T} B_{c} \\
\hat{A}_{c}^{i}=P_{1} A_{i} M_{1}+P_{1} B D_{c} C M_{1}+P_{2}^{T} B_{c} C M_{1} \\
+P_{1} B C_{c} M_{2}+P_{2}^{T} A_{c}^{i} M_{2}
\end{array}\right.
$$

one can obtain

$$
\sum_{i=1}^{4} h_{i}\left(\alpha_{f}, \alpha_{r}\right) \Upsilon_{i} \geq 0
$$

where

$$
\Upsilon_{i}=\left[\begin{array}{ccccc}
(1-\alpha) P_{1} & * & * & * & * \\
(1-\alpha) I & (1-\alpha) M_{1} & * & * & * \\
0 & 0 & \alpha Q & * & * \\
A_{i}+B \hat{D}_{c} C & A_{i} M_{1}+B \hat{C}_{c} & B \hat{D}_{c} D+E & M_{1} & * \\
P_{1} A_{i}+\hat{B}_{c} C & \hat{A}_{c}^{i} & \hat{B}_{c} D+P_{1} E & I & P_{1}
\end{array}\right]
$$

Notice that the matrices $M_{1}, M_{2}, P_{1}$ and $P_{2}$ verify:

$$
M_{2}^{T} P_{2}=I-M_{1} P_{1}
$$

In addition, it is possible to handle constraints on the control signal and the state:

$$
-\bar{u} \leq u(t) \leq \bar{u}, \quad-\bar{\Psi} \leq \Psi x(t+1) \leq \bar{\Psi}, \quad \forall t \geq 0
$$

where $\bar{u}>0, \bar{\Psi}:=\left[\bar{\Psi}_{1}, \ldots, \bar{\Psi}_{q}\right]^{T}$ with $\bar{\Psi}_{j}>0, j=1, \ldots, q, \Psi \in$ $\mathscr{R}^{q \times 4}$. Notice that the bounds are provided separately on each state variables or as combination of state variables.

For a pre-specified scalar $\eta \in(0,1]$, the quadratic boundedness property ensures that if $\tilde{x}(0) \in \varepsilon_{\eta P}$, then $\tilde{x}(t) \in \varepsilon_{\eta P}, \forall t \geq 0$, thus $\forall w(t) \in \varepsilon_{Q}$

$$
\begin{aligned}
& \max _{t \geq 0}\left|u(t)^{2}\right|=\max _{t \geq 0}\left|\left[D_{c} C C_{c}\right] \tilde{x}(t)+D_{c} D w(t)\right|^{2} \\
& \leq \max _{t \geq 0} \|\left[\left[\begin{array}{lll}
D_{c} C & \left.C_{c}\right] & D_{c} D
\end{array}\right]\left[\begin{array}{cc}
\eta P & 0 \\
0 & Q
\end{array}\right]^{-1 / 2} \|^{2}\right. \\
& \times\left\|\left[\begin{array}{cc}
\eta P & 0 \\
0 & Q
\end{array}\right]^{1 / 2}\left[\begin{array}{c}
\tilde{x}(t) \\
w(t)
\end{array}\right]\right\|^{2}
\end{aligned}
$$

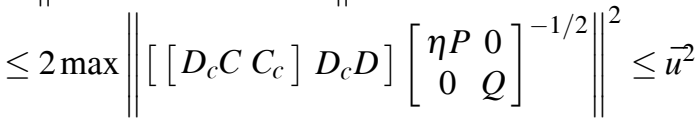

By applying the Schur complement, the condition (24) is satisfied if

$$
\left[\begin{array}{ccc}
\eta P & * & * \\
0 & Q & * \\
\sqrt{2}\left[D_{c} C C_{c}\right] & \sqrt{2} D_{c} D & \vec{u}^{2}
\end{array}\right] \geq 0
$$

By pre- and post-multiplying the left-hand side of the inequality (25) with $\operatorname{diag}\left\{T_{1}^{T}, I, I\right\}$ and $\operatorname{diag}\left\{T_{1}, I, I\right\}$, respectively, and then expanding $T_{1}$, one can obtain inequality:

$$
\left[\begin{array}{cccr}
\eta P_{1} & * & * & * \\
\eta I & \eta M_{1} & * & * \\
0 & 0 & Q & * \\
\sqrt{2} \hat{D}_{c} C & \sqrt{2} \hat{C}_{c} & \sqrt{2} \hat{D}_{c} D & \vec{u}^{2}
\end{array}\right] \geq 0
$$

A similar procedure can be applied for the constraints on the state variables. One can achieve from the convex property conditions (27):

$$
\begin{array}{ll}
\sum_{i=1}^{r} h_{i}\left(\alpha_{f}, \alpha_{r}\right) \widetilde{\Upsilon}_{i} \geq 0, & t \geq 0, \\
\Xi_{k k} \leq \Psi_{k}^{2}, & k \in\{1, \ldots, q\}
\end{array}
$$

where $\Xi$ is a symmetric matrix and

$$
\widetilde{\Upsilon}_{i}=\left[\begin{array}{cccc}
\eta P_{1} & * & * & * \\
\eta I & \eta M_{1} & * & * \\
0 & 0 & Q & * \\
\widetilde{\Upsilon}_{i 41} & \widetilde{\Upsilon}_{i_{42}} & \widetilde{\Upsilon}_{i_{43}} & \Xi
\end{array}\right]
$$

and

$$
\begin{gathered}
\widetilde{\Upsilon}_{i_{41}}=\sqrt{2} \Psi\left(A_{i}+B \hat{D}_{c} C\right) \\
\widetilde{\Upsilon}_{i_{42}}=\sqrt{2} \Psi\left(A_{i} M_{1}+B \hat{C}_{c}\right) \\
\widetilde{\Upsilon}_{i_{43}}=\sqrt{2} \Psi\left(B \hat{D}_{c} E+D\right)
\end{gathered}
$$

\section{Controller synthesis}

Under the proposed modeling approach, the desired yaw rate could be seen as an input disturbance under which the closed-loop system should remain stable with bounded values for the state vector components. More generally, the state variables should not exceed the bounds of a "safety zone", namely $\left|\alpha_{f}\right| \leq \alpha_{f}^{M},\left|\alpha_{r}\right| \leq \alpha_{r}^{M}$ and $\left|\delta_{f}\right| \leq \delta_{f}^{M}$. Thus, the state vector $x$ has to be confined to a hypercube $L\left(Z^{M}\right)$ defined by the above bounds. Finally, the control input, the steering angle rate and the yaw moment, have to be bounded $\left|\dot{\delta}_{f}\right| \leq \dot{\delta}_{f}^{M}$ and $\left|T_{z}\right| \leq T_{z}^{M}$. 
According to the equation (23), $\bar{u}=\left[\dot{\delta}_{f}^{M}, T_{z}^{M}\right]$, while $\bar{\Psi}=$ $\left[\alpha_{f}^{M}, \alpha_{r}^{M}, \delta_{f}^{M}\right]^{T}$ and

$$
\psi=\left[\begin{array}{ll}
I_{3} & 0
\end{array}\right]
$$

The PDC output feedback controller was synthesized with the following numerical values:

$$
\begin{array}{lll}
\alpha=0.02, & \eta=0.02, & \dot{\delta}_{f}^{M}=100 \mathrm{deg} / s \\
T_{z}^{M}=10 K N & \alpha_{f}^{M}=\alpha_{r}^{M}=13 \operatorname{deg} \delta_{f}^{M}=6 \mathrm{deg}
\end{array}
$$

These design parameters could be adjusted to handle the tradeoff between safety constraints and comfort specifications.

The achieved $Q$ is 5, which ensures that the constraints are verified for a disturbance of a magnitude less than $0.447 \mathrm{rad} / \mathrm{s}$ at the considered longitudinal speed of $20 \mathrm{~m} / \mathrm{s}$. In fact, the maximum value is constrained by the road adhesion coefficient $\mu$ and is given by [27]:

$$
r_{d_{\max }}=0.85 \mu \frac{g}{v}
$$

\section{SIMULATION TESTS}

In order to demonstrate the assistance ability to maintain the dynamic vehicle stability in extreme conditions, several type of maneuvers have been defined to test the ESC systems. Among them, in this paper are used the double lane-change manoeuvre defined in ISO 3888-2 standard and the "sine with dwell" transient maneuver considered by NHTSA (National Highway Traffic Safety Administration). The ISO388-2 setup is depicted in Figure 4-a with the installed cones and the track axis in dotted line. The maneuver is carried out with and without the controller at the same speed of $80 \mathrm{~km} / \mathrm{h}$ with the steering angle profile for the uncontrolled car shown in Figure 4-b in dashed line. During the maneuver, the throttle is released.

\section{Full adhesion situation}

While the road adhesion is at its nominal value, the driver initiates the double lane-change maneuver by applying the steering angle shown in dashed line in Figure 4-b. Figures 4-b and 5-a show that the controller shares the effort on the steering angle and the yaw moment (solid lines). In this situation the driver applied steering angle is too high (dashed line) while the the steering angle of the controlled vehicle is limited to the admissible safety value of few degrees while the yaw moment handles the main effort. Figure 5-b shows that the controlled car yaw rate is closer to the reference one (dash-dot line) than the yaw rate of the uncontrolled vehicle (dashed line). Figures 4-a highlights that the controlled vehicle is able to perform the maneuver (solid line) while the uncontrolled vehicle fails (dashed line). The contribution of each fuzzy sub-model to the vehicle dynamics are shown in Figure 6. Finally, Figures 7-a and 7-b provide the developed sideslip angles at the front and rear tires. The corresponding front and rear forces are shown in Figure 8 where a vertical translating factor is applied for the rear one for better visibility. It is clear that the saturation zones are reached during the maneuver.

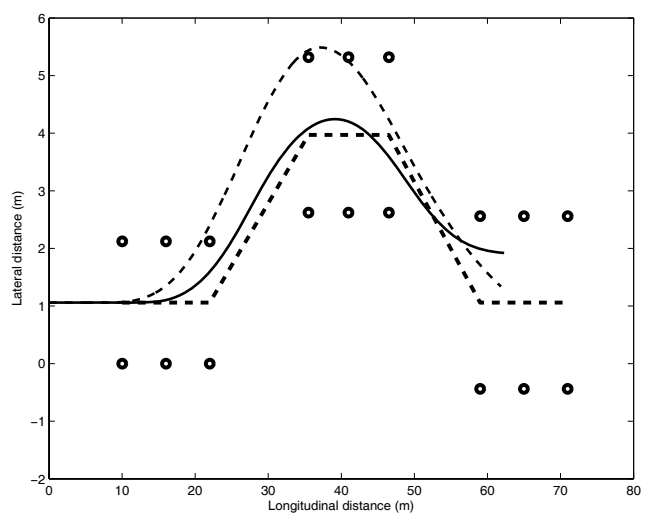

(a)

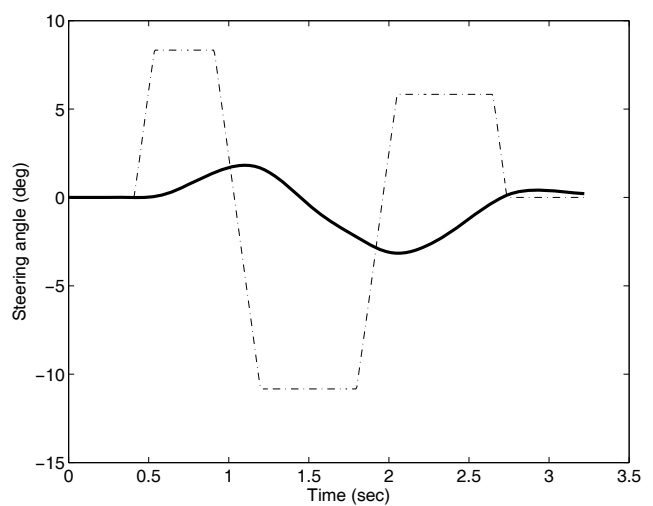

(b)

FIGURE 4. ISO3888-2 maneuver at nominal adhesion: Trajectory and steering angle for the uncontrolled and the controlled vehicles

\section{Reduced adhesion situation}

The same maneuver is now conducted with a road adhesion reduced to $\mu=0.75$. As can be seen form figures 9 and 10 , the controlled vehicle is still able to perform the maneuver but 

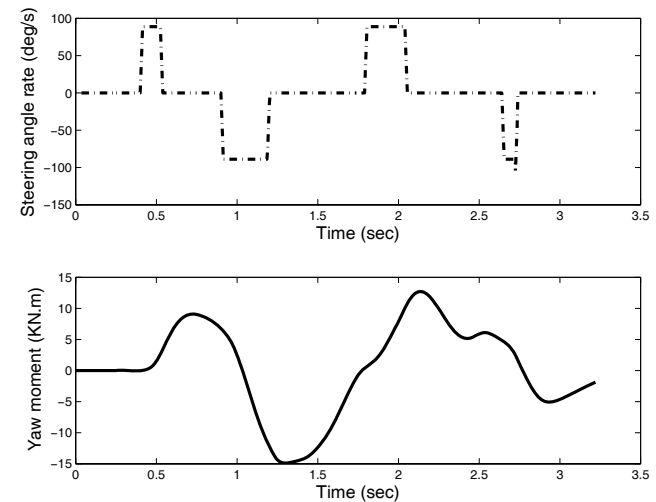

(a)

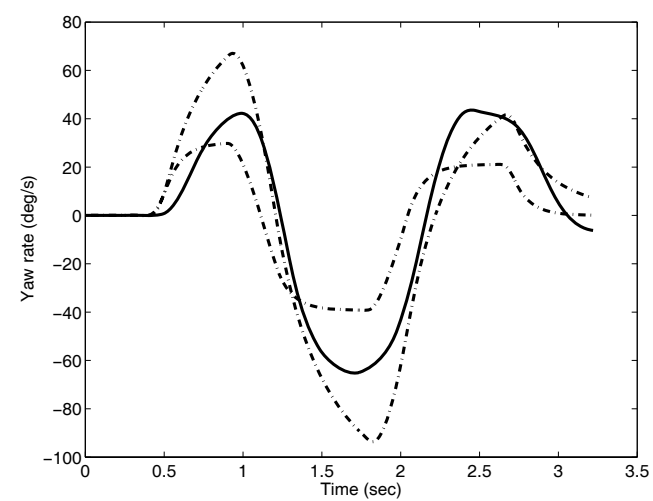

(b)

FIGURE 5. ISO3888-2 maneuver at nominal adhesion: Steering angle rate and yaw rate and for the uncontrolled and the controlled vehicles

with degraded performances. The behavior of the uncontrolled vehicle is still unsatisfactory.

\section{CONCLUSIONS AND FUTURE WORKS}

In this paper the design and the test of an integrated steering and differential yaw moment control has been described. The nonlinear behavior of the vehicle dynamics have been coped through Takagi-Sugeno modeling while an output feedback fuzzy PDC controller allows the development of a controller which ensures an ellipsoidal invariant set. This invariant set presents two main advantages: a bounded overshoot of the state variables with respect to potential disturbances. Simulation tests have shown that the controlled vehicle is able to achieve the ISO 3888-2 transient maneuver where the uncontrolled one fails. The controlled vehicle exhibits also a good yaw movement damping. The final paper will include further responses analysis for J-turn and "sine with dwell" maneuvers.

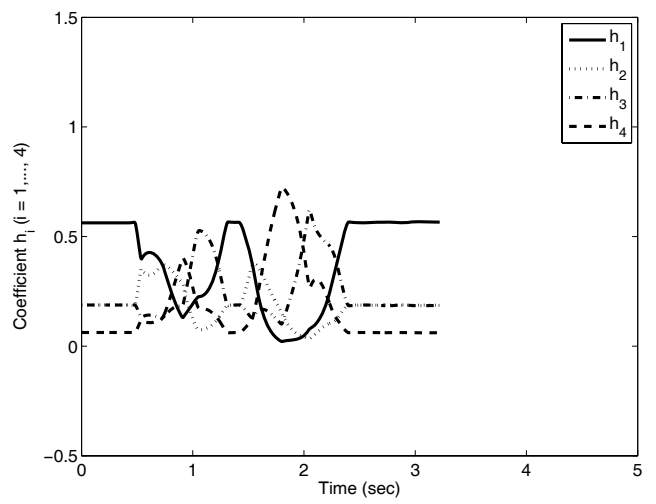

(a)

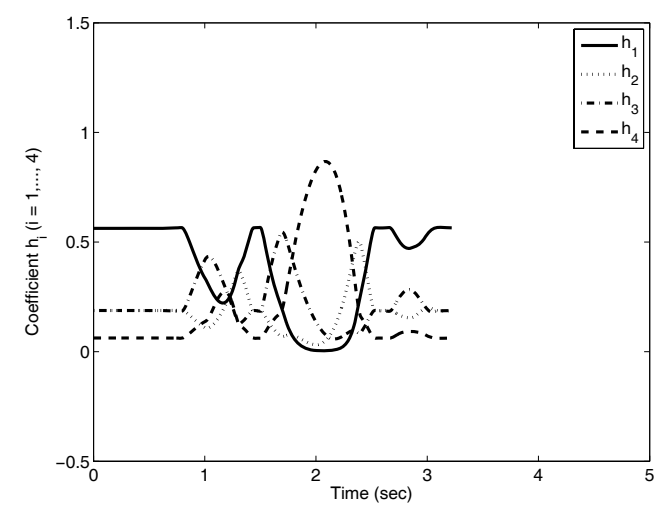

(b)

FIGURE 6. ISO3888-2 maneuver at nominal adhesion: Coefficients $h_{i}$ reflecting the contribution of each sub-model for the uncontrolled and the controlled vehicles

\section{REFERENCES}

[1] T. Takagi, M. Sugeno, Fuzzy identification of systems and its application to modeling and control, IEEE Trans. Systems Man Cybern. 15, pp. 116-132, 1985.

[2] A. Alessandri, M. Baglietto, G. Battistelli, On estimation error bounds for receding-horizon filters using quadratic boundedness. IEEE Transactions on Automatic Control, 49, 1350-1355, 2004.

[3] A. Alessandri, M. Baglietto, G. Battistelli, Design of state estimators for uncertain linear systems using quadratic boundedness. Automatica, 42, 497-502, 2006.

[4] B. Ding, Quadratic boundedness via dynamic output feedback for constrained nonlinear systems in Takagi-Sugeno's form, Automatica, vol. 45, N 9, pp. 2093-2098, 2009.

[5] C. H. Fang, Y. S. Liu, S. W. Kau, L. Hong, C. H. Lee, A new LMI-based approach to relaxed quadratic stabilization of T S fuzzy control systems. IEEE Transactions on Fuzzy Systems, 14, 386-397, 2006. 


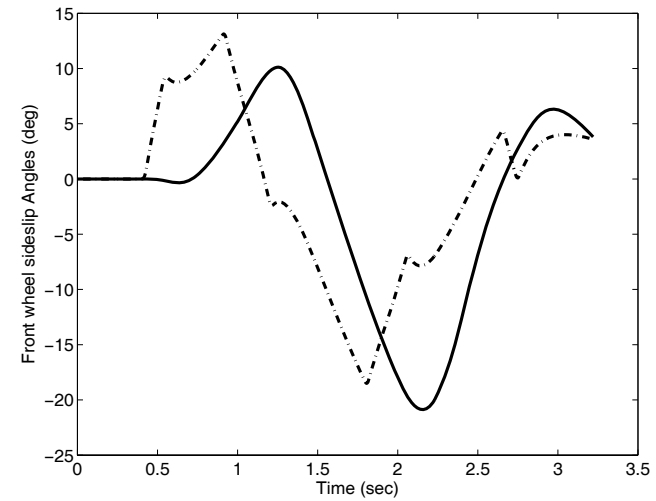

(a)

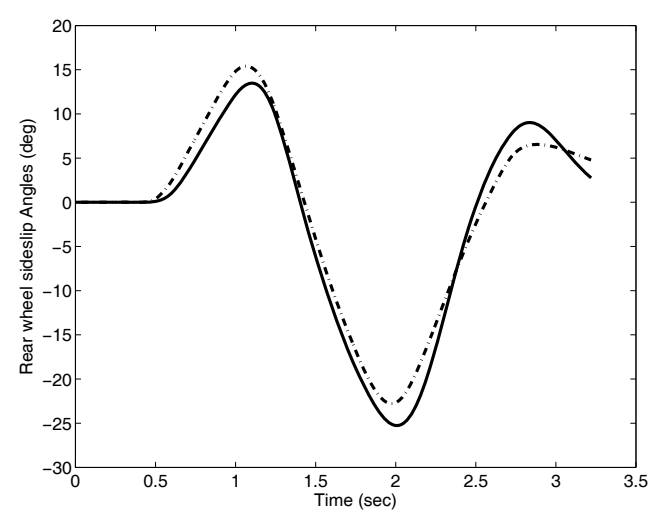

(b)

FIGURE 7. ISO3888-2 maneuver at nominal adhesion: Front and rear tire sideslip angle for the uncontrolled and the controlled vehicles

[6] G. Feng. Stability analysis of discrete-time fuzzy dynamic systems based on piecewise Lyapunov functions. IEEE Transactions on Fuzzy Systems, 12, 22-28, 2004.

[7] T.M. Guerra, L. Vermeiren, LMI-based relaxed nonquadratic stabilization conditions for nonlinear systems in the Takagi-Sugeno's form. Automatica, 40, 823-829, 2004.

[8] S. K. Kau, H. J. Lee, C. M. Yang, C. H. Lee, L. Hong, C. H.Fang, Robust H-infinity fuzzy static output feedback control of T-S fuzzy systems with parametric uncertainties. Fuzzy Sets and Systems, 158, 135-146, 2007.

[9] J. C. Gerdes et E. J. Rossetter A Unified Approach to Driver Assistance Systems Based on Artificial Potential Fields Authors. Journal of Dynamic Systems, Measurement and Control, Vol. 123, No. 3, pp. 431-438, 2001.

[10] X. D. Liu, \& Q. L. Zhang, New approaches to H-infinity controller designs based on fuzzy observers for T-S fuzzy systems via LMI. Automatica, 39, 1571-1582, 2003.

[11] H.O Wang, K. Tanaka, M. Griffin, An approach to fuzzy

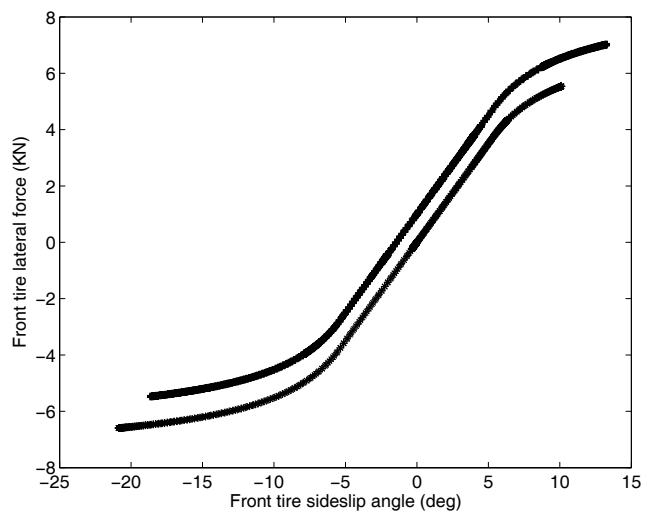

(a)

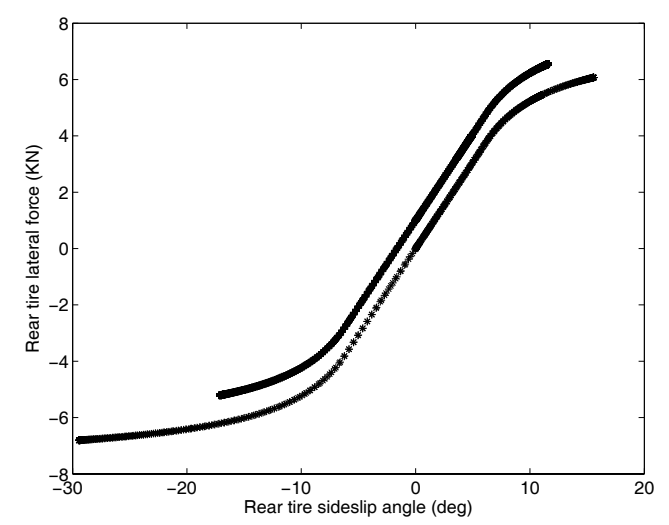

(b)

FIGURE 8. ISO3888-2 maneuver at nominal adhesion: Front and rear lateral forces for the uncontrolled and the controlled vehicles (with offset for the uncontrolled one)

control of nonlinear systems: Stability and design issues. IEEE Transactions on Fuzzy Systems, 4, 14-23, 1996.

[12] S. Mammar, T. Raharijaona, S. Glaser, G. Duc, Lateral driving assistance using robust control and embedded drivervehicle road model, Vehicle System Dynamics, Volume 41 Supplement, pp 311-320, 2004.

[13] E. Bakker, H. B. Pacejka, and L. Lidner. A new tire model with an application in vehicle dynamics studies. SAE paper, 1989.

[14] S. Mammar and D. Koenig, Vehicle Handling improvement by Active Steering, Vehicle System Dynamics Journal, vol 38, No3, pp. 211-242, 2002.

[15] K. Tanaka, H.O. Wang, Fuzzy Control Systems Design and Analysis, Wiley Inc, New York, 2001.

[16] N. Minoiu, M. Netto, S. Mammar, B. Lusetti, Driver steering assistance for lane departure avoidance, Control Engineering Practice, Vol. 17, No 6, pp. 642-651, 2009.

[17] H. Dugoff, P. Francher et L. Segel, An analysis of tire trac- 


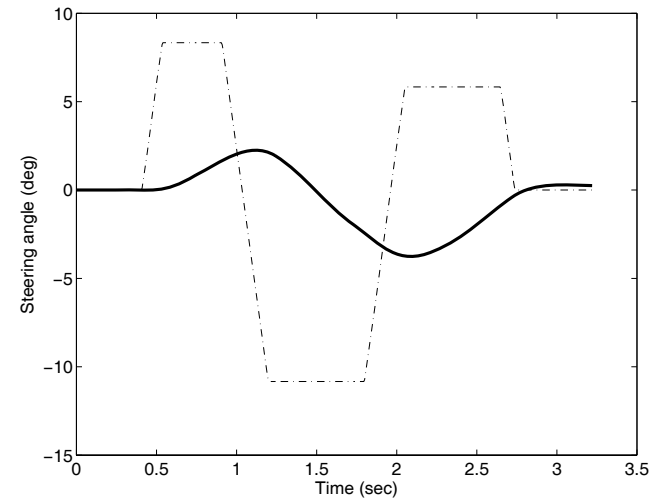

(a)
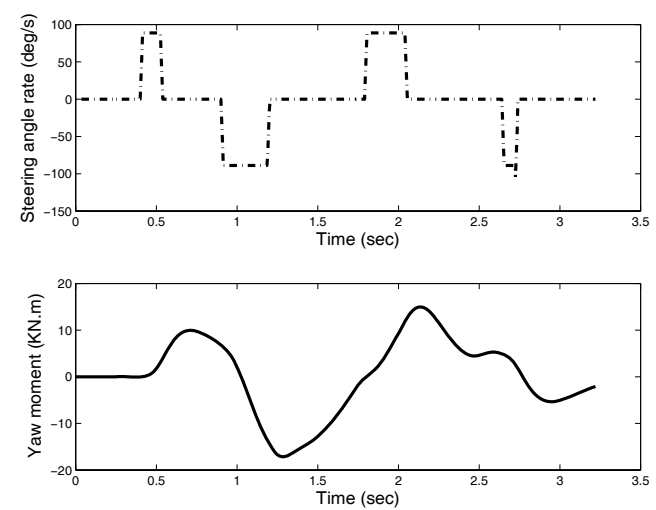

(b)

FIGURE 9. ISO3888-2 maneuver at reduced adhesion: Steering angle and steering angle rate for the uncontrolled and the controlled vehicles

tion properties and their influence on vehicle dynamic performance, SAE document $\mathrm{N}^{\circ}$ 700377, 1970.

[18] Grands thèmes de la sécurité routière en France. ONISR, Observatoire national interministériel de sécurité routière, février 2007.

[19] A.T.V. Zanten, R. Erhart, and G. Pfaff, VDC, The Vehicle Dynamics Control System of Bosch, , SAE Technical Paper No. $95759,1995$.

[20] A.T.V. Zanten, Bosch ESP Systems: 5 Years of Experience, SAE Technical Paper No. 2000-01-1633, 2000.

[21] M.J. Hancock, R.A. Williams, T.J. Gordon, M.C. Best, A comparison of braking and differential control of road vehicle yaw-sideslip dynamics, Proceedings of IMechE., Part D: Automobile Engineering, V.219, pp. 309-327, 2005.

[22] J. Tjoennas and T. A. Johansen, "Adaptive Optimizing Dynamic Control Allocation Algorithm for Yaw Stabilization of an Automotive Vehicle using Brakes,' in Control and Automation, 2006. MED '06. 14th Mediterranean Conference

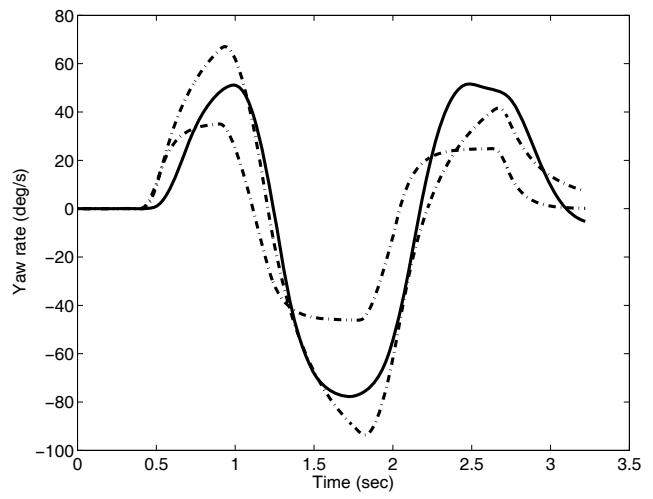

(a)

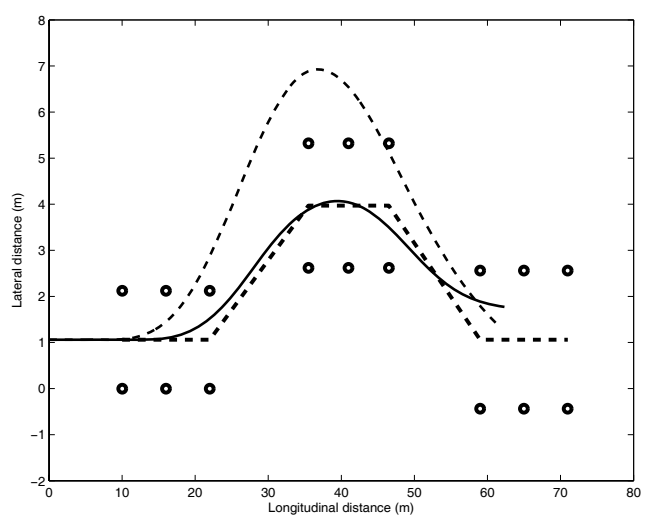

(b)

FIGURE 10. ISO3888-2 maneuver at reduced adhesion: Yaw rate and trajectory for the uncontrolled and the controlled vehicles

on, 2006, pp. 1-6.

[23] J. Ackermann, D. Odenthal and T. Bünte. Advantages of active steering for vehicle dynamics control., pp. 263-270, 1999.

[24] E. Ono, Y. Hattori, Y. Muragishi, K. Koibushi, Vehicle Dynamics Integrated Control for Four-Wheel-Distributed Steering and Four-Wheel-Distributed Traction/Braking Systems, Vehicle System Dynamics, V.44, No.2, pp. 139$151,2006$.

[25] Y. Shibahata, K. Shimada and T. Tomari, Improvement of vehicle maneuverability by direct yaw moment control, Vehicle Syst. Dynamics, vol 22, N 5-6, pp. 465-481, 1993

[26] P. Kohen and M. Ecrick, Active Steering - The BMW Approach Towards Modern Steering Technology, SAE Technical Paper No. 2004-01-1105, 2004.

[27] Rajamani R., Vehicle Dynamics and Control, Springer, New-York, 2006. 